\title{
Quasi-helical Symmetry at Finite Aspect Ratio
}

\author{
C. Nührenberg ${ }^{1}$, M.I. Mikhailov ${ }^{2}$, J. Nührenberg ${ }^{1}$, V.D. Shafranov ${ }^{2}$ \\ ${ }^{1}$ Max-Planck-Institut für Plasmaphysik, EURATOM Association \\ D-17491 Greifswald, Germany \\ ${ }^{2}$ Russian Research Centre "Kurchatov Institute", Moscow, Russia
}

Abstract. Computational stellarator optimization is used to create a configuration which is quasi-helically symmetric at finite aspect ratio. For the aspect ratio per period chosen $(\approx 2)$ this procedure results in benign properties throughout the plasma volume.

\section{Introduction}

Previously, stellarator optimizations of quasi-symmetric configurations have been performed with respect to the complete plasma volume, see e.g. $[1,2]$. Since it was on one hand proven that quasi-symmetries cannot be achieved exactly over the entire plasma volume and, on the other hand, that they can be strictly obtained on one flux surface [3], a different option for optimization is to choose a flux surface at finite aspect ratio and realize quasi-symmetry there. This option is investigated here: The boundary of the quasi-helically symmetric configuration obtained in [1] is selected and a 47-dimensional configurational space comprising boundary Fourier coefficients with poloidal mode numbers $m$ and toroidal mode numbers $|n|$ up to 3 is used to try to achieve quasi-helical symmetry at a toroidal aspect ratio $\approx 12$ corresponding to an aspect ratio per period of $\approx 2$. The result is the subject of this brief communication.

\section{Results}

Figure 1 shows structures of the strength of $B=\sum_{m n} B_{m n} \cos 2 \pi(m \theta+n \phi)$ [with $\theta$ and $\phi$ the poloidal and toroidal angle-like magnetic coordinates] in terms of its small Fourier components, $B_{m n}$ (the two largest components, $B_{00}$ and $B_{1-1}$, are not shown). While in the configuration of [1] all coefficients tend to get larger with increasing flux this is clearly the case only for the helically symmetric coefficients in the configuration obtained here; the coefficients perturbing the quasi-symmetry form two classes: those corresponding to the optimization space chosen exhibit the quasi-symmetry at the plasma boundary rather perfectly; the amplitudes of higher-order Fourier components not corresponding to the optimization space remain at the few per mill level.

The comparison of the two configurations seen in Fig. 2 shows that the flux surface geometry resulting from the optimization performed here is well-behaved. In cylindrical coordinates $(R, \varphi, Z)$, the plasma boundary is defined by the two functions $R(u, v)=$ $\sum_{m n} R_{m n} \cos 2 \pi(m u-n v)$ and $Z(u, v)=\sum_{m n} Z_{m n} \cos 2 \pi(m u-n v)$, where $v=N_{p} \varphi /(2 \pi)$, $N_{p}=6$, and $u$ a poloidal parametrization. The boundary coefficients, $R_{m n}$ and $Z_{m n}$, of the cases discussed here are given in Tables I and II. No constraints on the rotational 
transform and the magnetic well have been used; as a result, the rotational transform is slightly larger (see Fig. 3), the magnetic well slightly deeper than in the configuration of $[1]$.

In the context of quasi-symmetry the characterization of neoclassical transport properties is of particular relevance. Fig. 4 shows that the equivalent neoclassical ripple (characterizing the level of the so-called $1 / \nu$ transport, see, e.g., $[4,5]$ and, specifically, $\epsilon^{\frac{3}{2}}$ in [6]) is of similar smallness in both configurations. From Fig. 5, it is seen that the bootstrap current $[7,8]$ is similar, and from Fig. 6 that the collisionless loss of $\alpha$-particles is small, but a factor of about 2 smaller for particles started at half the plasma radius and a factor of about 4 smaller for particles started at 0.7 of the plasma radius in the configuration obtained here.

\section{Conclusion}

It appears that the procedure used here to obtain nearly quasi-symmetric configurations is viable, too. The computational optimization was performed with a NAG routine (E04UCF), i.e. exploiting the smoothness of the problem. A genetic algorithm [9] was used to verify the global nature of the optimum found. 


\section{Acknowledgment}

This work was supported in part by the Russian Foundation for Basic Research, project no 09-02-0142-a and by grant 2457.2008.2 of President of Russian Federation for state support of leading scientific school. 


\section{References}

[1] J. Nührenberg and R. Zille, Physics Letters A 129 1988, 113.

[2] J. Nührenberg et al, in Theory of Fusion Plasmas Editrice Compositori, Bologna 1994, 3 .

[3] D.A. Garren and A.H. Boozer, Phys. Fluids B 3 1991, 2805.

[4] A.A. Galeev and R.Z. Sagdeev, Zh. Eksp. Teor. Fiz. 53 1967, 348 [Sov. Phys. JETP 26 1968, 233].

[5] W. Lotz and J. Nührenberg, Phys. Fluids 31 1988, 2984.

[6] V.V. Nemov et al, Plasma Phys. Contr. Fusion 45 2003, 43.

[7] R.J. Bickerton, J.W. Connor, and J.B. Taylor, Nat. Phys. Sci. 239 1971, 110.

[8] W.A. Cooper et al, Plasma Phys. Contr. Fusion 44 2002, B357.

[9] A. Ben Haj-Yedder, in Automatic Differentiation of Algorithms: From Simulation to Optimization, edited by G. Corliss, C. Faure, A. Griewank and L. Hascoet (Springer, Berlin, 2002). 


\section{Figure captions}

Fig. 1. Small Fourier coefficients of the magnetic field strength in magnetic coordinates of two quasi-helical configurations, top from [1] and bottom obtained here; $B_{00} \approx 1.3$ and $B_{1-1} \approx-0.18$ at the boundary (top), $B_{00} \approx 1.4$ and $B_{1-1} \approx-0.22$ at the boundary (bottom) are not shown; — low-m coefficients perturbing the quasi-symmetry; - - - quasi-symmetric coefficients; $\times \times \times$ high-m coefficients perturbing the quasi-symmetry. The 17 largest of the small coefficients are shown.

Top: - $:(3,-2),(2,-3),(2,0),(0,-1),(1,1),(3,0),(1,-2),(2,-1) ;----:(3,-3)$, $(4,-4),(2,-2) ; \times \times \times:(5,-4),(4,-2),(4,-1),(7,-6),(6,-5),(4,-3)$.

Bottom: - : $(0,-1),(1,-2),(1,0),(2,-1),(2,-3),(1,-3),(3,-4) ;----:(3,-3)$, $(4,-4),(5,-5),(6,-6),(7,-7),(8,-8),(2,-2) ; \times \times \times:(5,-4),(4,-5),(7,-8)$.

Fig. 2. Magnetic surfaces at the beginning, quarter of and half of a period for two quasihelically symmetric configurations; top from [1], bottom obtained here. The boundary coefficients of the latter are given in Tables I and II.

Fig. 3 Rotational transform vs. flux label; solid line: configuration found here, dashdotted line: [1].

Fig. 4. Equivalent neoclassical ripple $\epsilon$ (here used in the form $\epsilon^{\frac{3}{2}}$ vs. flux surface label; solid line: configuration found here, dash-dotted line: [1]. The spike in $\epsilon$ from [1] is due to the incidental resonance, $\iota_{\text {period }}=\frac{1}{4}$.

Fig. 5. Structural factor of the bootstrap current vs. flux surface label; solid line: configuration found here, dash-dotted line: [1]. The difference in the structural factor at large flux label is again due to $\iota_{\text {period }}=\frac{1}{4}$ near the boundary.

Fig. 6. Four different loss histories of 1000 collisionless $\alpha$-particles started (randomly distributed in the angular variables and pitch angle) at half and 0.7 of the plasma radius. Normalization: plasma volume $10^{3} \mathrm{~m}^{3}$, magnetic field $5 \mathrm{~T}$. Each symbol marks the loss of one particle in a cumulative way. The straight lines indicate the fractions of reflected particles (in each case the lower line corresponds to half the plasma radius); dash-dotted lines, $\square$ and *: configuration found here; solid lines, $\circ$ and $\diamond$ : [1]. 
Tables

\begin{tabular}{rrrlr}
\hline $\mathrm{n}$ & $m$ & & & \\
\cline { 2 - 5 } & 0 & 1 & 2 & \multicolumn{1}{c}{3} \\
\hline-3 & 0 & 0.0026 & 0.0003 & -0.0008 \\
-2 & 0 & -0.0027 & -0.01 & 0.0011 \\
-1 & 0 & 0.0404 & -0.006 & -0.0071 \\
0 & 11 & 1.1761 & 0.0463 & -0.0317 \\
1 & 0.6833 & -0.5672 & 0.242 & 0.0315 \\
2 & 0.0214 & -0.0765 & 0.0665 & -0.0752 \\
3 & 0.0019 & 0.0058 & 0.0322 & -0.0017 \\
\hline
\end{tabular}

Table I. $R$ boundary coefficients of a 6-periodic case optimized for quasihelical symmetry on the plasma boundary.

\begin{tabular}{rlrrl}
\hline $\mathrm{n}$ & $m$ & & & \\
\cline { 2 - 5 } & 0 & 1 & 2 & 3 \\
\hline-3 & 0 & 0.0001 & 0.0003 & -0.0006 \\
-2 & 0 & -0.0025 & -0.0081 & -0.0003 \\
-1 & 0 & 0.033 & -0.0062 & -0.0049 \\
0 & 0 & 0.8239 & 0.0547 & -0.0144 \\
1 & -0.8546 & 0.3713 & 0.2141 & -0.0007 \\
2 & -0.0242 & 0.0791 & -0.0244 & -0.0177 \\
3 & -0.0074 & 0.0012 & -0.025 & -0.012 \\
\hline
\end{tabular}

Table II. Z boundary coefficients of a 6-periodic case optimized for quasihelical symmetry on the plasma boundary. 

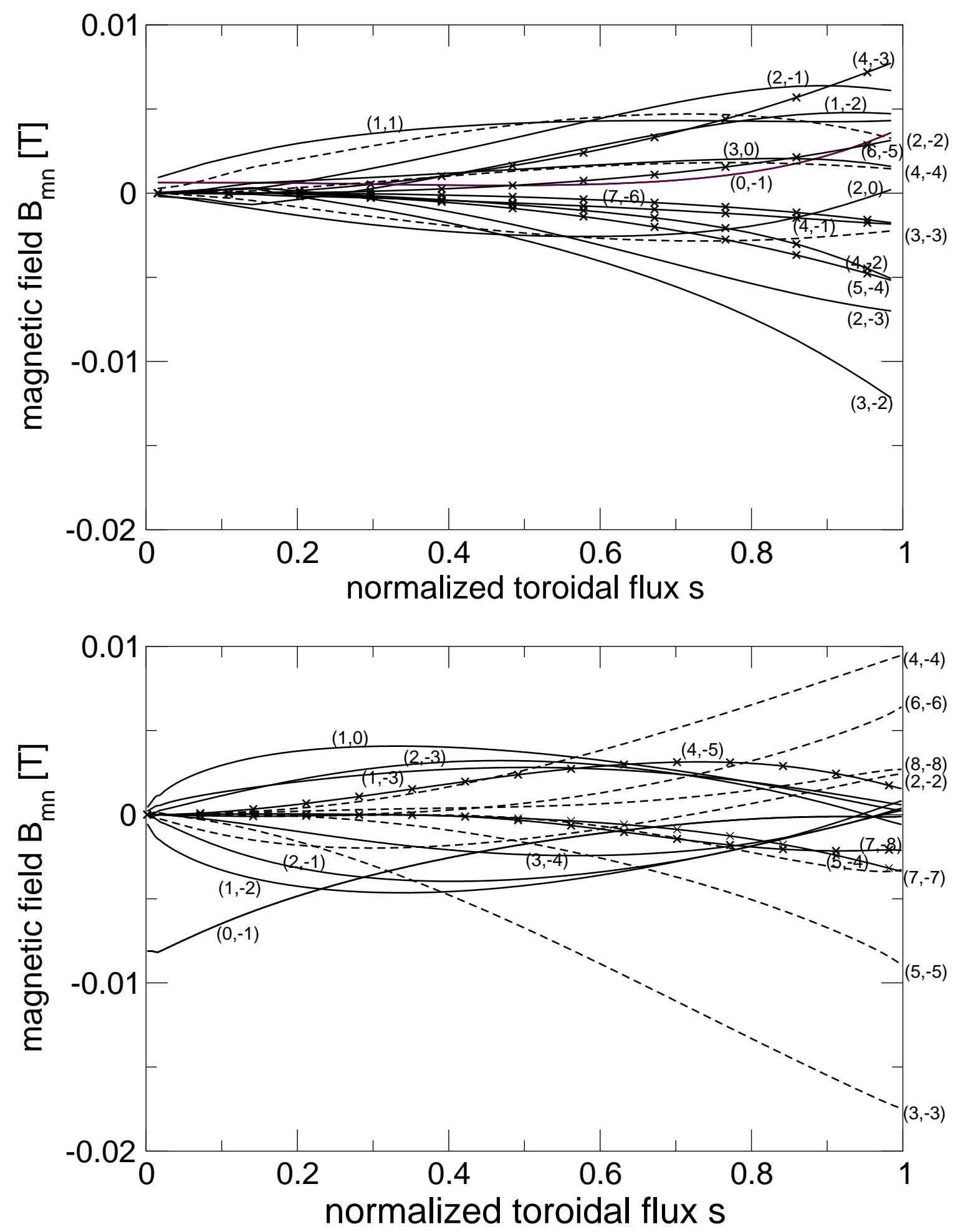

Figure 1 

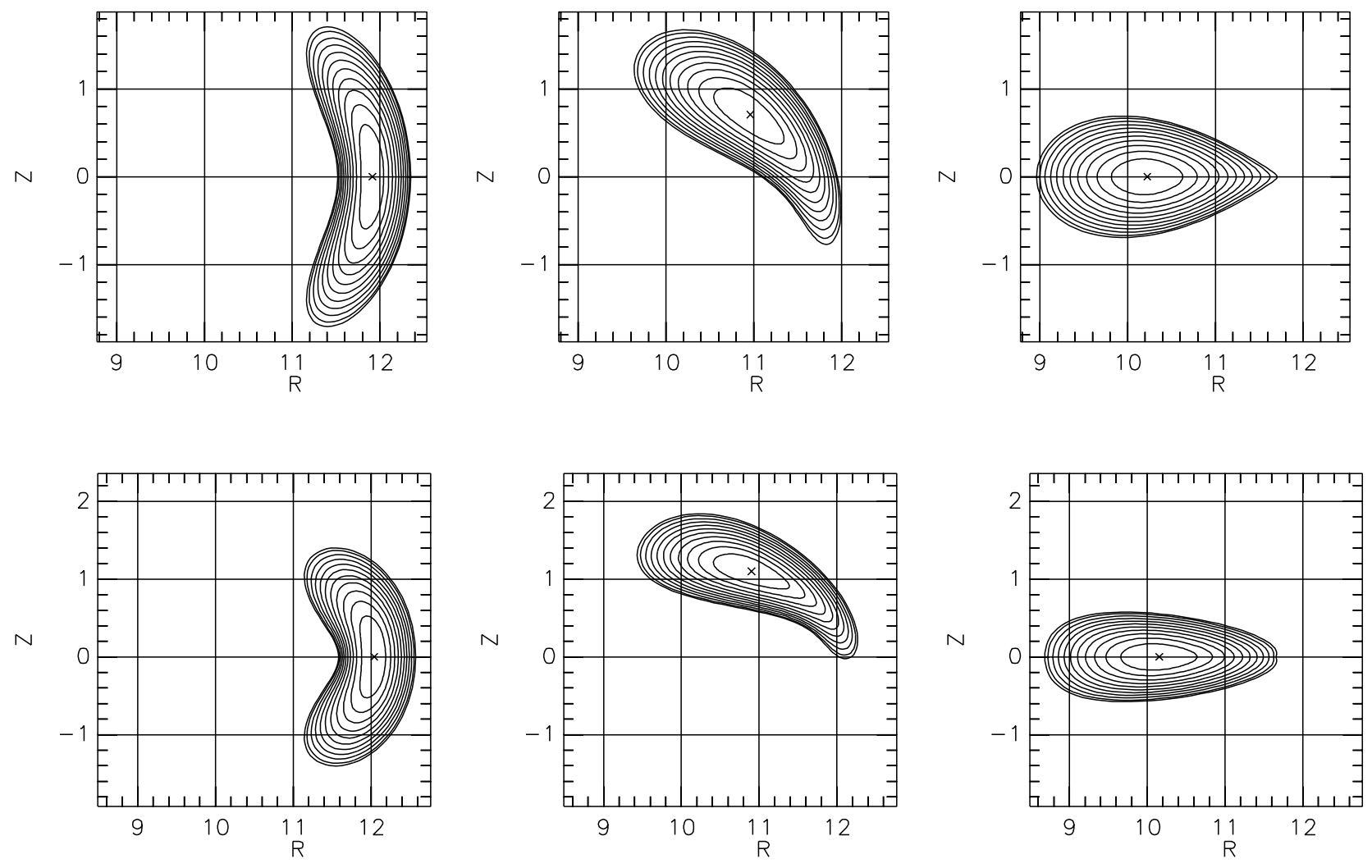

Figure 2 


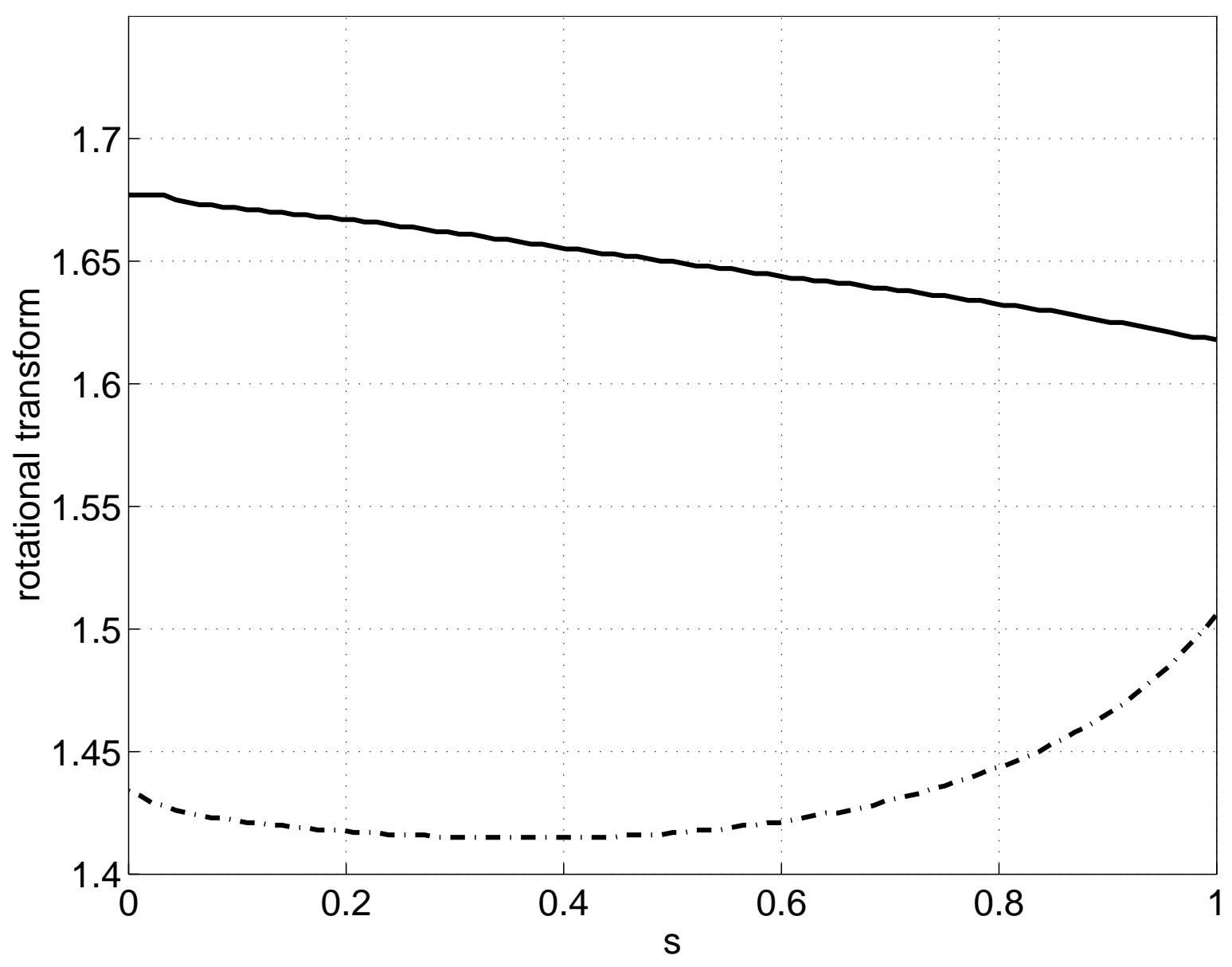

Figure 3 


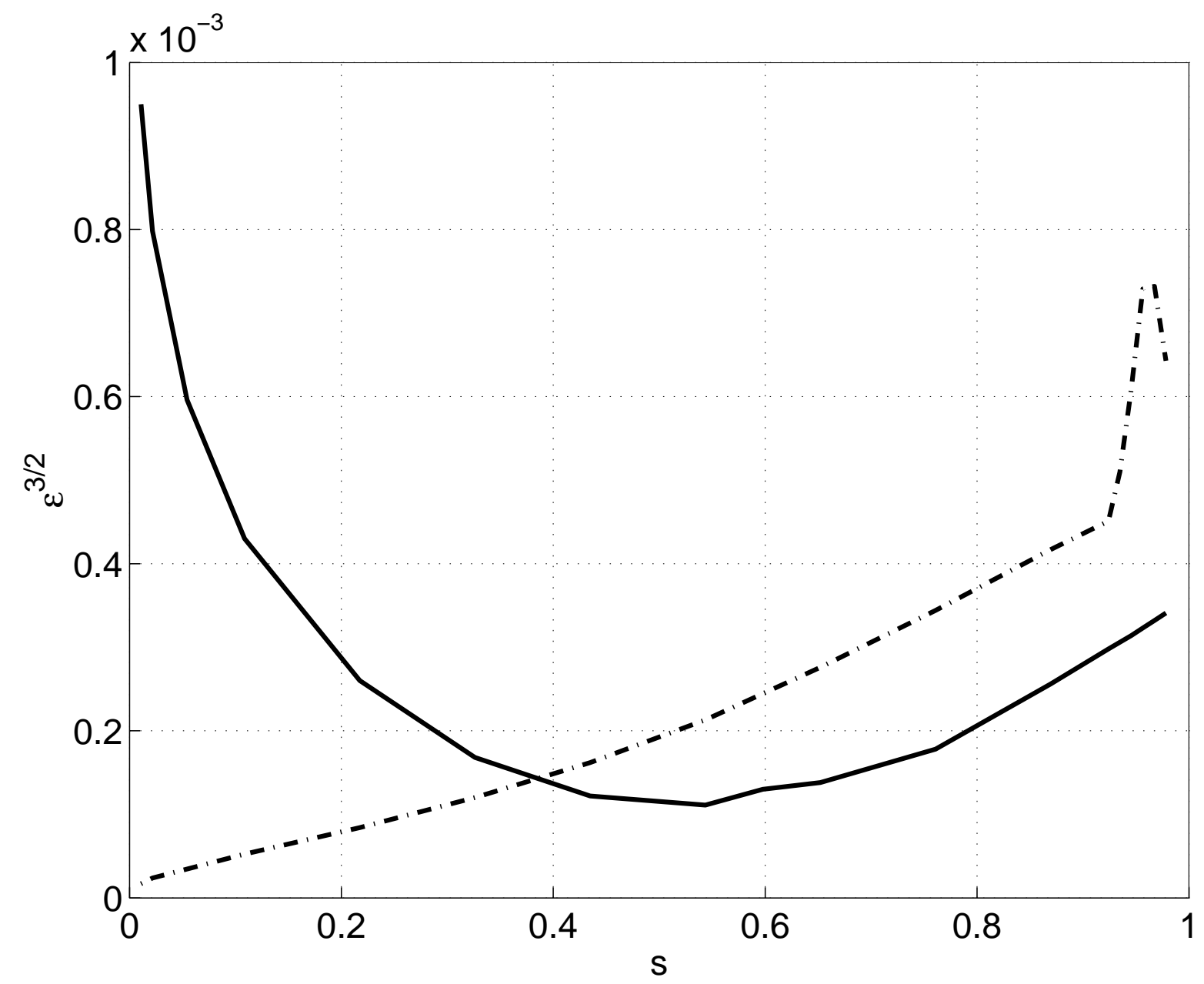

Figure 4 


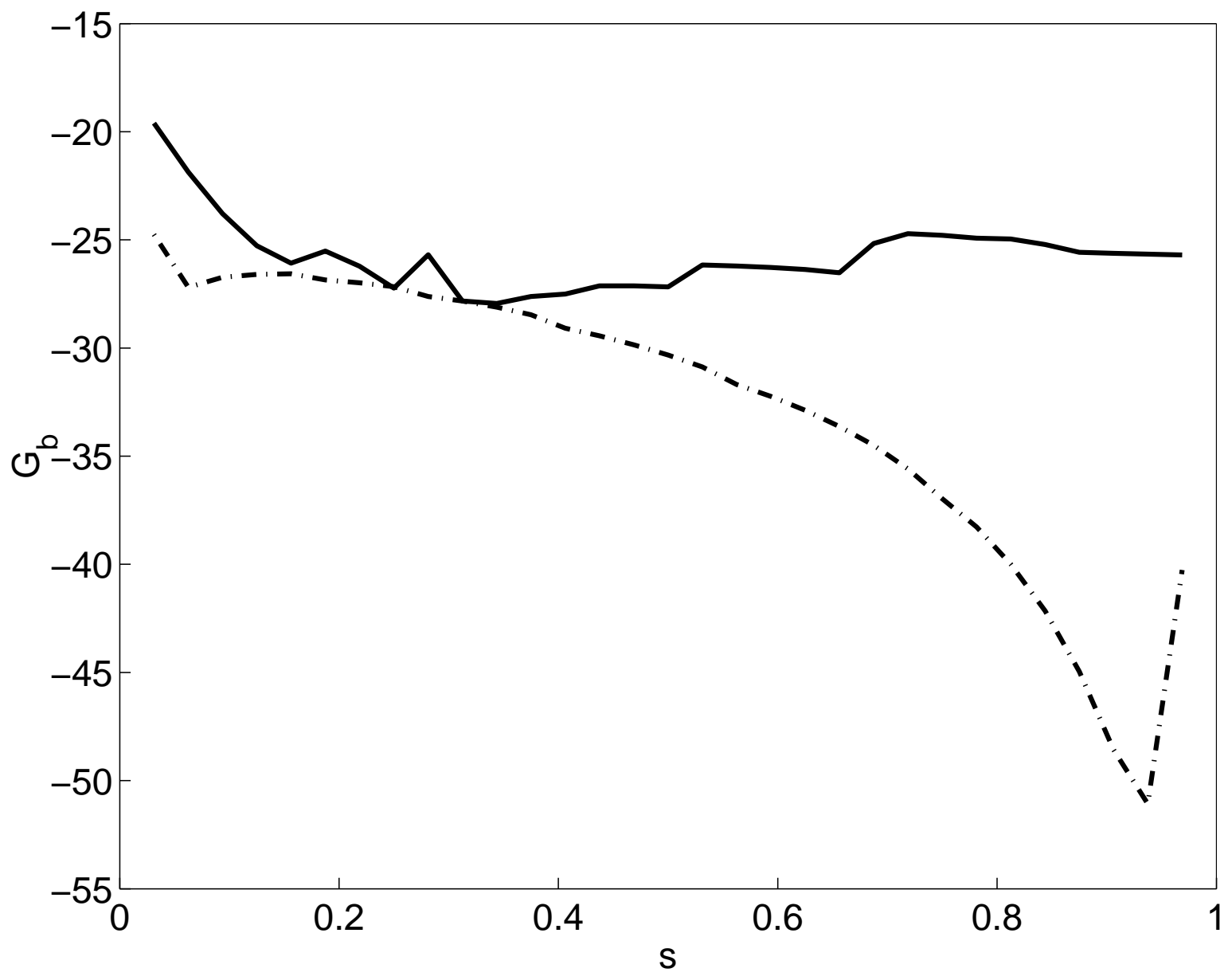

Figure 5 


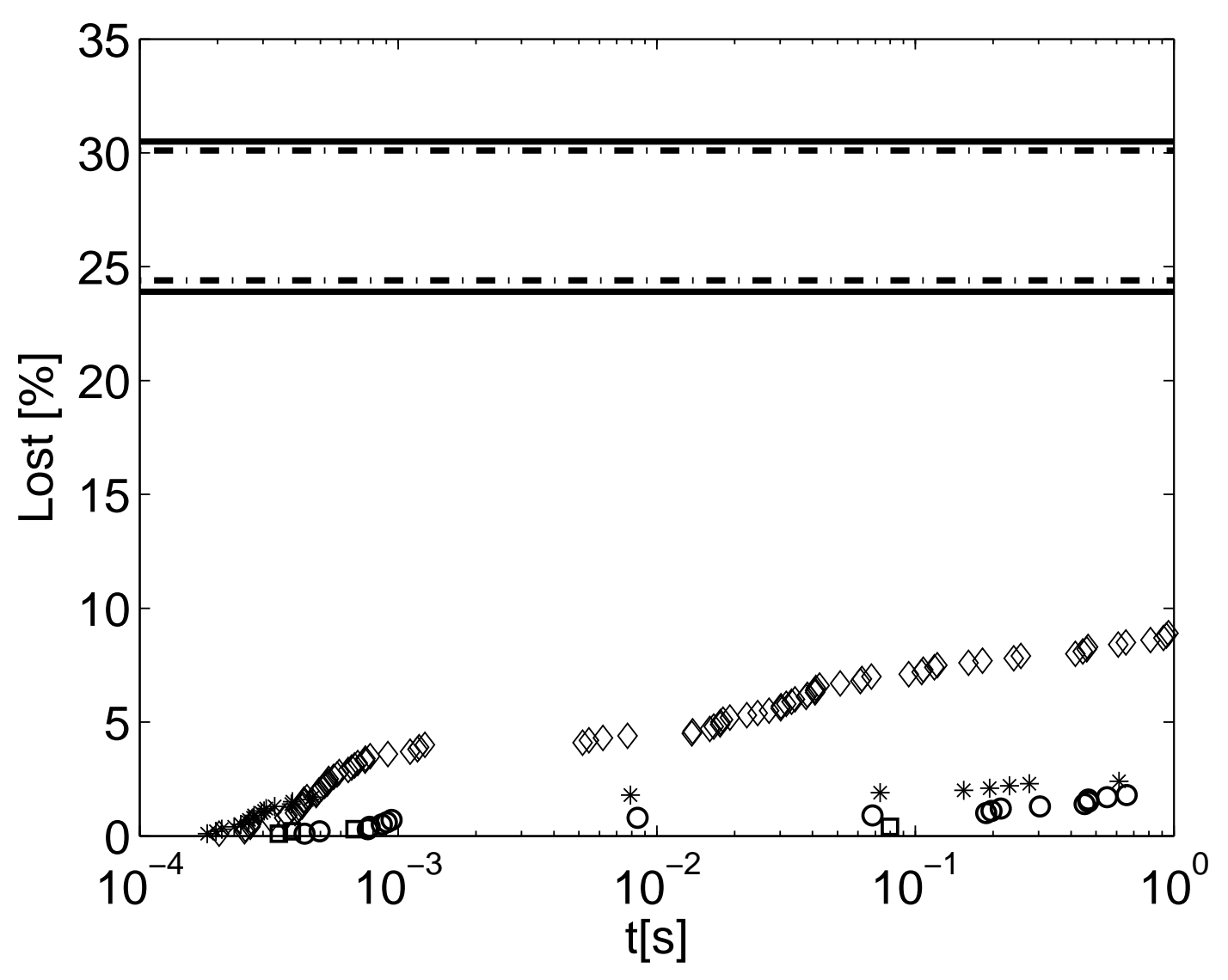

Figure 6 